\title{
BMJ Open Examining Australian's beliefs, misconceptions and sources of information for COVID-19: a national online survey
}

\author{
Rae Thomas (D) , ${ }^{1}$ Hannah Greenwood, ${ }^{1}$ Zoe A Michaleff (D) , ${ }^{1}$ Eman Abukmail, ${ }^{1}$ \\ Tammy C Hoffmann (D) , ${ }^{1}$ Kirsten McCaffery (D) , ${ }^{2}$ Leah Hardiman, ${ }^{3}$ \\ Paul Glasziou (i) ${ }^{1}$
}

To cite: Thomas $\mathrm{R}$, Greenwood H, Michaleff ZA, et al. Examining Australian's beliefs, misconceptions and sources of information for COVID-19: a national online survey. BMJ Open 2021;11:e043421. doi:10.1136/ bmjopen-2020-043421

- Prepublication history and additional material for this paper is available online. To view these files, please visit the journal online (http://dx.doi.org/10. 1136/bmjopen-2020-043421).

Received 05 August 2020 Revised 15 November 2020 Accepted 02 February 2021

\section{Check for updates}

\section{(c) Author(s) (or their} employer(s)) 2021. Re-use permitted under CC BY-NC. No commercial re-use. See rights and permissions. Published by BMJ.

${ }^{1}$ Institute for Evidence-Based Healthcare, Faculty of Health Sciences and Medicine, Bond University, Gold Coast, Queensland, Australia

${ }^{2}$ Sydney Health Literacy Lab, Sydney School of Public Health, Faculty of Medicine and Health, University of Sydney, Sydney, New South Wales, Australia ${ }^{3}$ Consumer Representative, Maternity Choices Australia, Brisbane, Queensland, Australia

Correspondence to

Dr Rae Thomas;

rthomas@bond.edu.au

\section{ABSTRACT}

Objective Public cooperation to practise preventive health behaviours is essential to manage the transmission of infectious diseases such as COVID-19. We aimed to investigate beliefs about COVID-19 diagnosis, transmission and prevention that have the potential to impact the uptake of recommended public health strategies.

Design An online cross-sectional survey.

Participants A national sample of 1500 Australian adults with representative quotas for age and gender provided by an online panel provider.

Main outcome measure Proportion of participants with correct/incorrect knowledge of COVID-19 preventive behaviours and reasons for misconceptions.

Results Of the 1802 potential participants contacted, 289 did not qualify, 13 declined and 1500 participated in the survey (response rate 83\%). Most participants correctly identified 'washing your hands regularly with soap and water' $(92 \%)$ and 'staying at least $1.5 \mathrm{~m}$ away from others' (90\%) could help prevent COVID-19. Over 40\% (incorrectly) considered wearing gloves outside of the home would prevent them from contracting COVID-19. Views about face masks were divided. Only $66 \%$ of participants correctly identified that 'regular use of antibiotics' would not prevent COVID-19.

Most participants (90\%) identified 'fever, fatigue and cough' as indicators of COVID-19. However, $42 \%$ of participants thought that being unable to 'hold your breath for $10 \mathrm{~s}$ without coughing' was an indicator of having the virus. The most frequently reported sources of COVID-19 information were commercial television channels (56\%), the Australian Broadcasting Corporation (43\%) and the Australian Government COVID-19 information app (31\%).

Conclusions Public messaging about hand hygiene and physical distancing to prevent transmission appears to have been effective. However, there are clear, identified barriers for many individuals that have the potential to impede uptake or maintenance of these behaviours in the long term. We need to develop public health messages that harness these barriers to improve future cooperation. Ensuring adherence to these interventions is critical.
Strengths and limitations of this study

- Participants in this survey are a nationally representative sample of Australian adults surveyed for COVID-19 knowledge and misconceptions.

- The survey content was informed by two separate search strategies for common COVID-19 misconceptions.

- Beliefs about misconceptions were qualitatively coded to determine common themes.

- Self-report data have limitations and biases which should be considered in the interpretation and implications of our findings.

- We did not examine the impact of health literacy, and cultural and linguistic diversity on people's beliefs which may impact the generalisability of our findings.

\section{INTRODUCTION}

So far, Australia has done well in its response to COVID-19, but not because of vaccines or drug treatments. The success is due to the use of non-drug interventions, such as physical distancing, hand hygiene and quarantining. ${ }^{1}$ Maintaining the suppression of major outbreaks is critical but it is predicated on garnering public cooperation to continuously conduct appropriate public health behaviours. In the absence of a vaccine, social cohesion and cooperation to practise COVID-safe behaviours is critical. ${ }^{2}$ Misinformation about prevention, transmission and treatment of COVID-19 has the potential to derail these efforts. ${ }^{3}$

Misinformation often fuels misconceptions. Misconceptions about COVID-19, particularly as the disease emerged, have been circulating in the media and online, prompting the WHO to construct a 'Mythbusters' webpage. ${ }^{3}$ Understanding the reasoning behind misconceptions and exploring typical sources 
of information can facilitate behaviour change interventions designed to support risk reduction efforts. ${ }^{4-6}$ Several surveys have explored people's perceptions, knowledge and attitudes of COVID-19. International and Australian-focused online surveys suggest $71 \%-93 \%$ of participants ${ }^{78}$ could identify preventive behaviours (eg, hand hygiene, physical distancing), but the proportion of individuals who self-report practising these behaviours were fewer, ranging from a low of $45 \%$ in a survey of UK participants ${ }^{9}$ to $85 \%$ in an Australian sample. ${ }^{10}$ The difference between the high proportion of people knowing appropriate prevention behaviours and the lower proportion of those reporting to practise the behaviour suggests that knowledge alone is insufficient to change behaviour. This constitutes a knowledge-practice gap. Varying demographic differences have been reported to predict practising preventive behaviours including low socioeconomic status, ${ }^{9}$ low health literacy, ${ }^{7}$ anxiety ${ }^{710}$ and those with high compared with low engagement and perceptions of risk. ${ }^{1011}$

The knowledge-practice gap identified in previous surveys requires exploration. To achieve high compliance with infectious disease prevention measures relevant to the current COVID-19 pandemic and future infectious outbreaks, we need to understand the reasoning behind why some people do not practise effective prevention behaviours. ${ }^{4}$ Therefore, this survey aimed to (1) identify misconceptions about COVID-19 that have the potential to impact the uptake of the appropriate prevention behaviours (particularly the public health messages from the Australian Government), (2) examine reasons why individuals hold misconceptions for key prevention strategies and (3) identify the most commonly used sources for acquiring COVID-19-related information.

\section{METHODS}

\section{Study design and participants}

A cross-sectional, online survey of eligible adult Australians was conducted from 8 May to 11 May 2020. The national sample, with representative quotas for age and gender, was provided by online panel provider, Dynata (https:// www.dynata.com/). The online survey was scripted using Qualtrics (https://www.qualtrics.com/au/).

People aged 18 years and older and living in Australia were eligible to participate. Healthcare professionals and people who had been tested for COVID-19 (which was not widely available at the time of the survey) were ineligible to participate as their knowledge about COVID-19 may differ compared with a more general Australian public. Prior to commencing the survey, potential participants read a detailed study explanatory statement. Continuation of survey was accepted as informed consent.

\section{Patient and public involvement}

A member of our research team and coauthor (LH) is a consumer representative and provided advice on common misconceptions identified in her role as consumer advocate.
These assisted in developing the search for misconceptions to inform the survey content. We also pilot tested the survey with members of the public external to our research.

\section{Survey}

To identify common misconceptions, we conducted two searches to identify public misconceptions that, if believed, would have the potential to negatively impact the uptake of the behaviours recommended by public health authorities. First, we conducted a focused literature search of PubMed for misconceptions from the current COVID-19 pandemic and previous epidemics (ie, keyword search included terms for severe acute respiratory syndrome, H1N1 influenza, Middle East respiratory syndrome, coronavirus and myths or misconceptions). Our second search was an environmental scan of internet and social media sites (eg, Facebook) using the terms 'COVID-19' and 'myths' (a term used by the $\mathrm{WHO}$ ) to identify colloquial misconceptions not represented in the formal literature. We also conducted citation checks of identified articles (research publications and internet/news articles) to detect further misconceptions and sources of information.

Each misconception identified in our dual search strategy was collated and the following information extracted for each. First, we recorded whether the misconception was found through a single or multiple source. Then, each misconception was categorised as relating to diagnosis, transmission and prevention of COVID19. These two steps were conducted by authors RT and HG. Next, each author rated the identified misconceptions as to whether believing that misconception had the potential to influence the prevention or transmission of COVID-19. We were only interested in including these misconceptions in the survey, as we considered these potentially harmful to public health. Disagreements were resolved through consensus.

For example, the misconception that 'keeping your mouth and throat moist' would prevent COVID-19 was identified in multiple social media platforms. This was categorised as a misconception about preventing COVID-19. However, we categorised this misconception as one that, if believed and enacted, would not negatively impact the prevention or transmission of the virus, and was therefore not included in the survey. Conversely, the misconception that 'taking antibiotics would prevent COVID-19' was reported from multiple sources. It was considered a misconception that, if believed, would impact prevention or transmission of COVID-19. For example, if someone takes antibiotics as they believe it protects them from COVID19 , this could potentially increase COVID-19 transmission in the community if they have the virus but believe they are protected (or cured). Hence, this misconception was included in the survey.

The study team included psychologists, medical practitioners and clinical epidemiologists. The survey was piloted with colleagues (including people who have English as a second language) and members of the 
public to ensure face and content validity, and ease of completion.

\section{Measures}

The survey consisted of five sections (COVID-19 symptoms, prevention strategies, beliefs for 'incorrect' prevention strategies, transmission behaviours and information sources for COVID-19). The survey including items, logic, and response scales are reported in online supplemental table 1 . The expected time to complete the survey was less than $10 \mathrm{~min}$.

\section{Statistical analysis}

The primary outcome was the frequency of prevention strategy misconceptions and participant reasoning for each. Descriptive statistics (counts and percentages) were calculated for participant demographic variables and correct/ incorrect survey responses. 'Correct' and 'incorrect' answers for survey questions were determined by the study team via group consensus a priori. With a sample size of 1500 , the margin of error around a $50 \%$ proportion is $\pm 2.5 \%{ }^{12}$ This sample size is similar to most national opinion polls which generally use samples in the range of 1000-2000.

For the open response options, we conducted content analyses $^{13}$ of the 'incorrect' responses. Two authors (RT and $\mathrm{LH}$ ) independently coded the first 50 responses and developed an initial coding framework. Uninformative responses (eg, 'I don't know'; 'unsure') were not coded. Codes were compared and the framework was iteratively developed until consensus was reached. The same two authors tested the refined coding framework on the next 20 responses. Following consensus, one author (RT) completed the qualitative analyses for the remaining responses. Due to the unexpectedly large volume of 'incorrect' responses for three items (wearing masks, wearing gloves and staying away from healthcare centres), a random $50 \%$ of the responses was coded. All responses were coded for the other four items (use of colloidal silver and essential oils, use of antibiotics, social distancing, washing hands).

\section{RESULTS}

Of the 1802 potential participants contacted, 289 were screened as ineligible prior to completing the survey and were excluded at that point, 13 declined and 1500 participated in the survey (response rate 83\%; see figure 1 for participant flow chart). As per the sampling frame, $50 \%$ of survey participants were female, there was a representation across adult age groups and a proportional representation from each Australian state and territory. Participant characteristics are reported in table 1 .

\section{Identifying prevention strategies}

A high proportion of the sample gave correct responses for hand hygiene and $1.5 \mathrm{~m}$ physical distancing, but significant proportions were incorrect for the effectiveness of antibiotics, colloidal silver, wearing gloves or masks, and

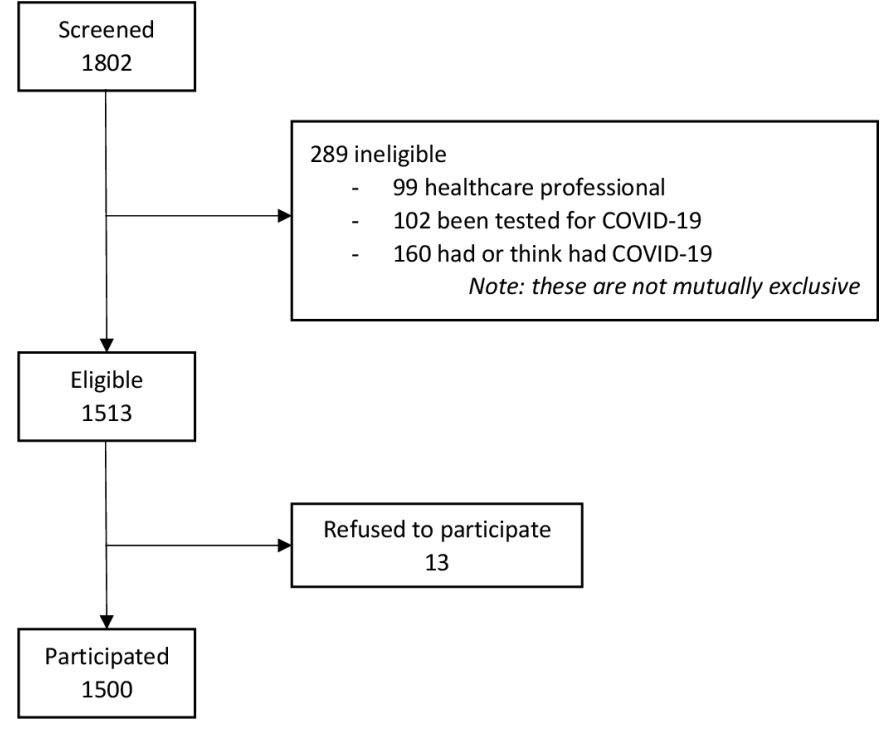

Figure 1 Participant flow chart.

staying away from health centres. Figure 2 shows proportions of participant perceptions of preventive behaviours and table 2 reports the results of the content analyses for the 'incorrect' responses.

Although most participants correctly identified 'washing your hands regularly with soap and water' $(92 \%), 7 \%$ were undecided ('neither agree or disagree' or 'I don't know') and a minority (2\%) thought that washing their hands would NOT prevent COVID-19. Reasons varied, but the most frequent theme $(37.5 \%)$ was that participants thought handwashing was unnecessary or ineffective (eg, it was an 'overreaction', it would not 'kill' the virus, or it is redundant because the participant did not leave the house; see table 2).

Similarly, $90 \%$ of participants correctly identified that 'staying at least $1.5 \mathrm{~m}$ away from others' could prevent COVID-19. Only 34 (2\%) participants thought that physical distancing would NOT prevent COVID-19 transmission. Half of those who held that belief reasoned that the strategy was ineffective or unnecessary (eg, 'stays in the air' and 'can float a lot further than that'; see table 2).

Fewer participants correctly identified that 'regular use of antibiotics' (66\%) would NOT prevent COVID-19, and $22 \%$ were unsure. For those who thought that antibiotics could prevent COVID-19 (11\%), two dominant themes emerged: an incorrect understanding of antibiotics and treatment options for COVID-19 (eg, they could 'block viruses', and 'prevent' or 'kill' the virus) and erroneously thinking antibiotics would strengthen their immunity.

While $56 \%$ of participants correctly identified that taking 'products like colloidal silver or essential oils' would NOT prevent a person from contracting COVID$19 \%$ and $32 \%$ were unsure, and the remainder thought those products would help to prevent COVID-19. Approximately $32 \%$ of those who thought colloidal silver and essential oils would prevent COVID-19 appeared to incorrectly understand the transmission process and prevention strategies for COVID-19. For example, 


\begin{tabular}{|c|c|c|}
\hline Characteristics & $\mathbf{N}$ & $\%$ \\
\hline Female & 750 & 50 \\
\hline \multicolumn{3}{|l|}{ Age } \\
\hline $18-24$ years & 171 & 11 \\
\hline 25-34 years & 264 & 18 \\
\hline $35-44$ years & 239 & 16 \\
\hline $45-54$ years & 223 & 15 \\
\hline $55-64$ years & 222 & 15 \\
\hline $65-74$ years & 227 & 15 \\
\hline 75 or older & 154 & 10 \\
\hline \multicolumn{3}{|l|}{ Education } \\
\hline High school graduate or less & 459 & 31 \\
\hline Trade certificate (I-IV) & 276 & 18 \\
\hline Tertiary & 765 & 51 \\
\hline \multicolumn{3}{|l|}{ Australian states and territories } \\
\hline Queensland & 302 & 20 \\
\hline New South Wales & 471 & 31 \\
\hline Australian Capital Territory & 29 & 2 \\
\hline Nothern Territory & 9 & 1 \\
\hline Western Australia & 160 & 11 \\
\hline Victoria & 382 & 25 \\
\hline Tasmania & 34 & 2 \\
\hline South Australia & 113 & 7 \\
\hline \multicolumn{3}{|l|}{ Aboriginal or Torres Strait Islander } \\
\hline Yes & 17 & 1 \\
\hline No & 1471 & 98 \\
\hline Prefer not to say & 12 & 1 \\
\hline Born in Australia & 1049 & 70 \\
\hline
\end{tabular}

participants reasoned these products would help because they were 'good in disinfecting', suggesting transmission only by touch and 'evidence that colloidal silver is a virus killer' and the products would 'kill the germs'.

The two contentious preventative measures of wearing gloves or wearing surgical masks when outside of the home showed conflicting beliefs about the benefits of these behaviours to prevent COVID-19. Over $40 \%$ of participants considered wearing gloves outside of the home would help prevent them from contracting COVID19. Their reasons were nuanced. In the randomly coded sample of $50 \%(\mathrm{n}=299), 33 \%$ of participants incorrectly understood the transmission of COVID-19 (eg, 'won't come into contact with the virus', 'stop transmission'). However, 23\% identified plausible self-protection reasons (eg, 'would not be touching surfaces where it might be on and then touching my face and rubbing my eyes with bare hands', 'reduces risk of uptake of pathogen from hands, which in turn reduces risk of transmission from hands to eyes, nose, mouth, etc').

For 'wearing of face masks outside of the home', $28 \%$ of participants agreed that this would NOT prevent them (the wearer) from contracting COVID-19, while $42 \%$ of participants thought this behaviour was preventative, and the remainder unsure. Within the randomly coded sample, the most frequently provided reason for wearing surgical masks (33\%) was an incorrect understanding of COVID-19 transmission (eg, 'because the virus cannot move via a surgical mask', 'you can't catch it'). Yet, almost a quarter (23\%) identified plausible reasons for wearing masks ('may prevent inhaling droplets from the air which may contain the COVID-19 virus', 'it is some form of barrier to my respiratory system').

Most participants agreed (58\%) or were unsure (28\%) that staying away from hospitals and health centres would prevent COVID-19 transmissions. Of the randomly coded responses, 52\% articulated concerns of infection from test-positive COVID-19 patients and staff, while $13 \%$ had reasoned that hospitals and health centres should be avoided in general (eg, 'hospitals are hotbeds of disease').

\section{Understanding transmission process}

When asked to identify ways in which they were likely or unlikely to contract COVID-19, participant responses were again varied (figure 3 ).

The majority of participants correctly identified that transmission of COVID-19 was likely to occur through 'sneezing/coughing' (92\%), 'touching a contaminated surface and then touching your eyes, nose or mouth' $(91 \%)$ and 'being physically near someone with COVID-19 for a long period of time' (90\%). Fewer participants $(63 \%)$ correctly identified that 'being near someone who had recently been overseas' made it more likely that they could contract COVID-19. Most participants also correctly identified that you are unlikely to contract COVID-19 from 'mosquito bite' (78\%) or by 'touching a package that had come from China' (64\%).

In contrast, $65 \%$ of participants thought it was very likely or likely they would contract COVID-19 due to 'being in a large room with someone for a short period of time' and $51 \%$ thought they had a very likely or likely chance by 'sitting on a surface where someone with COVID-19 sat'. In a similar response pattern to preventing transmission, there was a lot of uncertainty about whether 'attending a doctor's clinic or hospital' increased your risk of contracting COVID-19 with an even proportion of participants thinking it was unlikely this would increase their risk $(48 \%)$ and those who thought it would $(40 \%)$ or were unsure $(12 \%)$.

\section{Awareness of COVID-19 symptoms}

The majority of participants $(90 \%)$ identified 'fever, fatigue and cough' as indicators of COVID-19 and which, if experienced, would require a person to take further precautions (table 3). However, just over a third of participants (35\%) correctly identified 'fever, fatigue and cough' as the only indicator (online supplemental table 
Q1\&2 Agree = Correct response.

From left to right: Strongly agree; Agree; Strongly disagree; Disagree; Neither; I don't know

\section{Q1. Washing your hands with soap regularly, especially before and after using the bathroom, eating, or leaving and reentering your home \\ Q2. Staying at least $\mathbf{1 . 5}$ metres away from people who you don't live with}

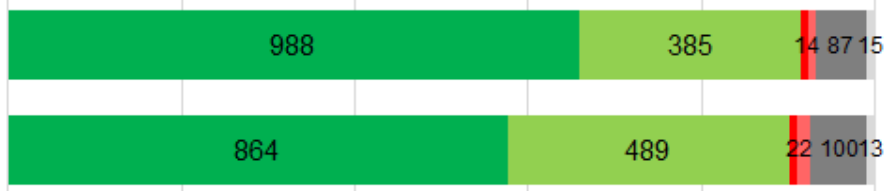

Q3-7 Disagree $=$ Correct response. From left to right: Strongly disagree; Disagree; Strongly agree; Agree; Neither; I don't know

Q3. Taking antibiotics regularly

Q4. Using products like colloidal silver or essential oils

Q5. Wearing gloves when you are away from your home

\section{Q6. Wearing a surgical mask when you are away from} your home

Q7. Staying away from hospitals and health centres

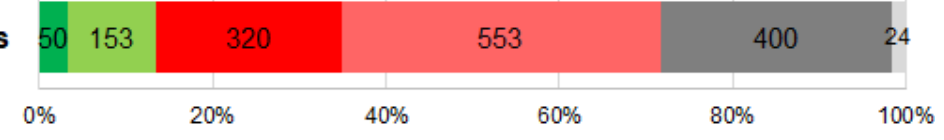

$\square$ Correct $\square$ Incorrect $\square$ Neither agree nor disagree $\square$ I don't know
2). Of concern, $42 \%$ of participants thought that being unable to 'hold your breath for $10 \mathrm{~s}$ without coughing' was an indicator of having COVID-19 (table 3).

\section{Information sources}

The most frequently reported sources of COVID-19 information were Channels 7, 9 and 10 news (56\%), ABC news (43\%), and the Australian Government COVID-19 app (31\%). Online supplemental table 3 shows the frequency in which each source of information was ranked as first, second or third. Only $23 \%$ of participants reported social media as a top 3 source of information and of those did, less than a quarter $(24 \%)$ of these ranked it as their first source of information.

\section{DISCUSSION}

To prevent COVID-19 infections, community members will have to sustain long-term preventive behaviours. To encourage community cooperation, public health officials will need to understand why some individuals do not engage with public health messaging and do not practise COVID-safe preventive behaviours. Therefore, this study had three aims: (1) identify misconceptions about COVID-19 that have the potential to inhibit the uptake of preventive behaviours; (2) examine reasons why individuals hold misconceptions for prevention strategies; and (3) identify the most commonly used sources for acquiring COVID-19-related information. The openended responses in this survey give unique information about why individuals hold inaccurate beliefs about preventive behaviours.

The widespread ability to identify the preventative measures of hand hygiene and physical distancing suggests that public health messaging has been effective in communicating prevention strategies to Australians. Although only a minority of participants thought these behaviours were unnecessary or ineffective, this was the major theme arising for people not practising either behaviour. Not knowing this reason for lack of cooperation is a missed opportunity. Using the Capability, Opportunity, Motivation- Behaviour change model (COM-B), ${ }^{14}$ these behaviours suggest of a lack of motivation and can be directly targeted through public health campaigns.

Almost all participants correctly identified key public health messaging of common symptoms of fever, fatigue and cough and potential transmission practices such as sneezing and coughing and touching contaminated surfaces. This prevention awareness is supported by several other surveys, with between $71 \%$ and $93 \%{ }^{710}$ of participants endorsing correct responses or reporting practising the behaviour.

However, when we examined preventative behaviours not explicitly communicated by public health authorities, the results are more varied. While two-thirds of participants correctly identified that taking antibiotics would not prevent COVID-19, the remaining third thought taking them would prevent COVID-19 or were unsure. Of concern, this result is similar to another 


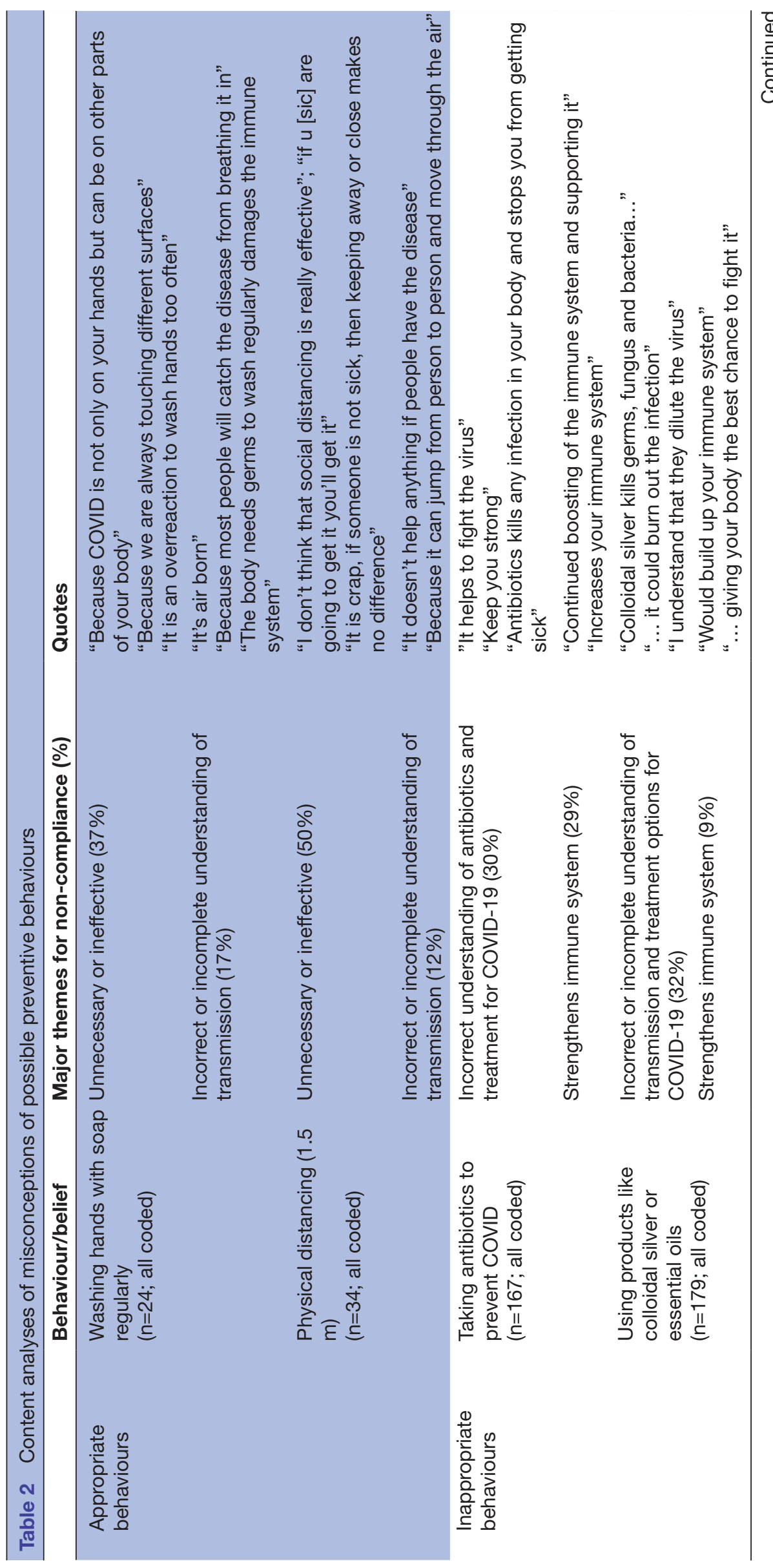




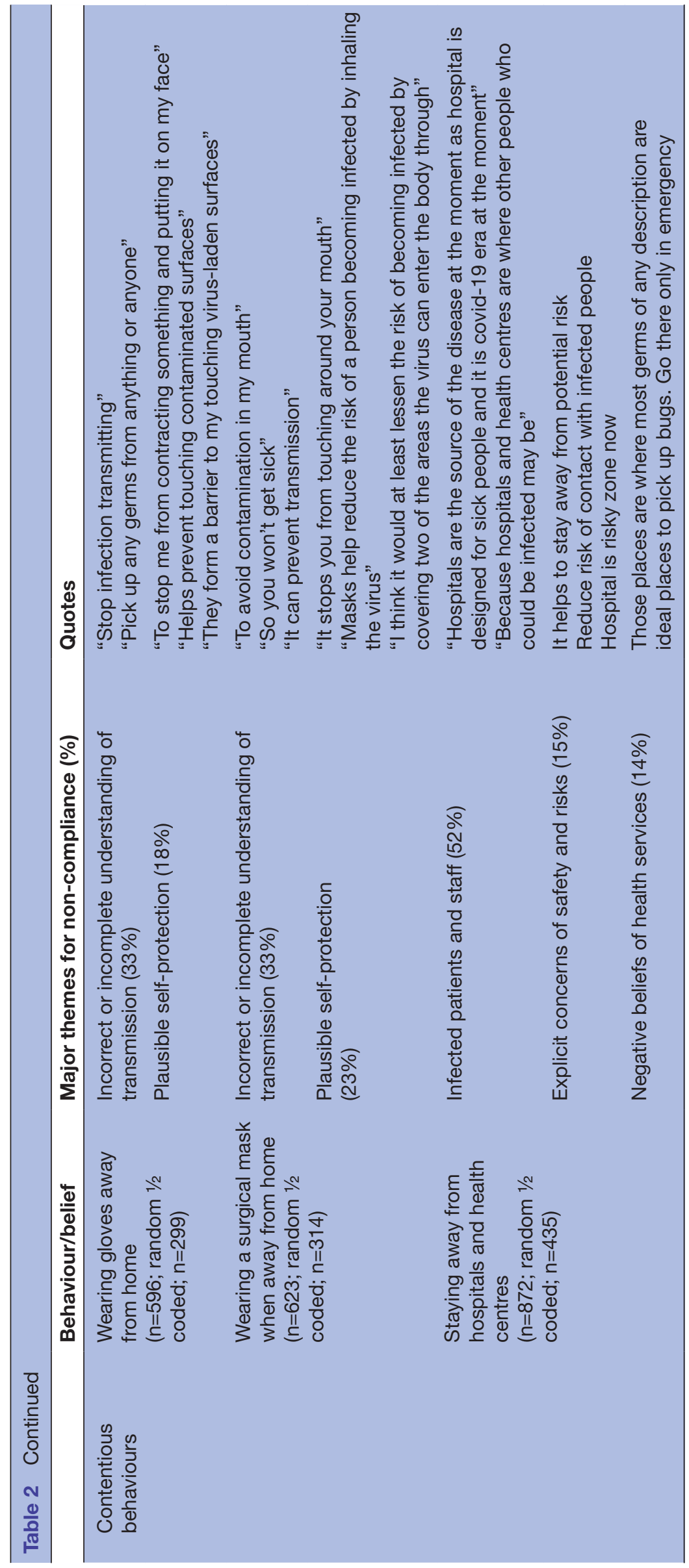


Q1-4 Very likely/likely to get COVID = Correct response.

From left to right: Very likely; Likely; Very Unlikely; Unlikely; I don't know

Q1. Someone with COVID-19 sneezing or coughing directly on you

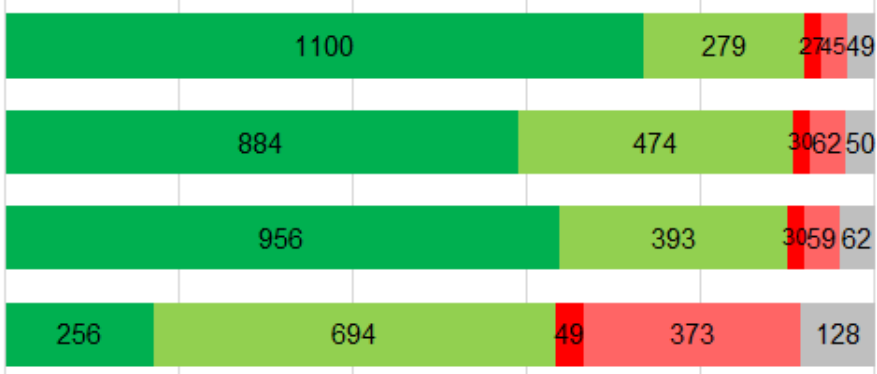

Q2. Touching a contaminated surface, then touching your eyes, nose or mouth

Q3. Being physically near someone who has COVID-19 for a long period of time

Q4. Being near or talking to someone who has been overseas recently

Q5-9 Very unlikely/unlikely to get COVID = Correct response. From left to right: Very unlikely; Unlikely; Very Likely; Likely; I don't know

Q5. Being bitten by a mosquito

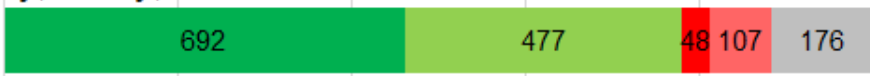

Q6. Touching a mail package that comes from China

Q7. Being at a doctor's clinic or hospital

Q8. Sitting on a surface where someone with COVID-19 sat

Q9. Being in a large room with someone who has COVID-19 for about 5 minutes

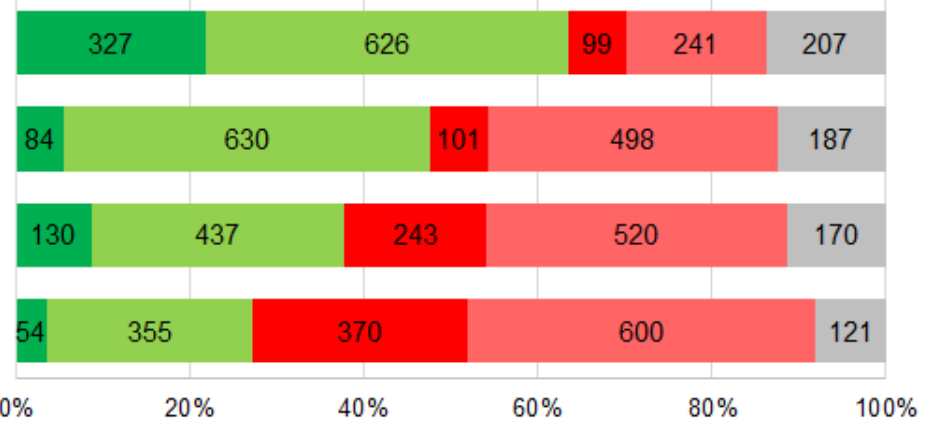

- Correct a Incorrect I don't know

Figure 3 Participant perceptions of their likelihood to contract COVID-19.

Australian study that reported $35 \%$ of participants also thought antibiotics would be effective to prevent or treat COVID-19. ${ }^{10}$ When asked for reasons for this misconception, it emerged that most who endorsed antibiotics had poor understanding of how they worked and their effects on viruses and the immune system broadly. Inappropriate use of antibiotics is considered the leading cause of antibiotic resistance. ${ }^{15} 16$ The perception that antibiotics could prevent or treat COVID-19 has the potential to derail ongoing efforts to curb antibiotic use in the community. ${ }^{17}$ Further, only half of participants correctly identified that products like colloidal silver or essential oils are not effective in preventing the transmission of COVID-19. Taken together, it appears that many Australians have misconceptions about the effectiveness

Table 3 Frequency of incorrect symptom/behaviour responses

\begin{tabular}{ll}
\hline Response & N (\%) \\
\hline $\begin{array}{l}\text { You cannot hold your breath for 10s without } \\
\text { coughing }\end{array}$ & $625(42)$ \\
$\begin{array}{l}\text { You have a runny nose and wet cough } \\
\text { You have had your temperature checked and it is }\end{array}$ & $234(16)$ \\
normal & $167(11)$ \\
\hline
\end{tabular}

Note: Participants were able to choose more than one response. of pharmaceutical and complementary medicine's roles in preventing COVID-19. Using behaviour change theory, this suggests a knowledge barrier that can be modified through tailored education and messaging using behaviour change techniques such as information about individual, social and environmental consequences. ${ }^{14}$ If people believe these approaches will stop them from contracting the virus, there is legitimate concern they will not practise non-drug interventions such as hand hygiene and physical distancing either now or in the future.

Equally concerning was our finding that at the time of the survey (early May), only $15 \%$ of participants were confident that visiting hospitals and health centres were safe. Australian data from the Medical Benefits Scheme ${ }^{18}$ appear to reflect this uncertainty, with a $23 \%$ drop in face-to-face primary care attendance compared with the average of the same month in the past 5 years. A sharp decline in presentations to emergency departments for reasons other than COVID-19 (eg, stroke, cardiac events and so on) has also occurred internationally. ${ }^{19-21}$ Conversely, the increase in telemedicine (eg, video and phone consultations) in Australia ${ }^{18}$ may suggest that although people are physically staying away from attending health services, they are still receiving healthcare through other means. These findings suggest that the emergence of COVID-19 has resulted in significant changes in how healthcare is used. The impact of these 
changes in terms of accessing preventative healthcare services, patient's health outcomes and healthcare costs is yet to be determined. ${ }^{22}$

Two preventative behaviours that were associated with a high degree of uncertainty were wearing gloves and wearing a mask outside of the home. Approximately $40 \%$ of participants believed wearing gloves or a mask would prevent transmission of COVID-19, similar to Seale et $a l$ s findings ${ }^{10}$ that $50 \%$ of surveyed Australians thought their use could prevent COVID-19. This uncertainty surrounding wearing a mask is likely a reflection of the mixed messages that were evident in the media and various public health campaigns used in both Australia and internationally during the early stages of the pandemic. These inconsistent and unclear messages may have contributed to the misconceptions about the effectiveness of masks and how they can reduce the transmission of COVID-19. Although wearing face masks might provide an added benefit to limit the spread of the infection, there has been no public health messages in Australia suggesting wearing gloves is an effective preventive behaviour. While our understanding of virus transmission is still developing, it is likely that, as a respiratory virus, a face mask alone is not sufficient to prevent its transmission. This was not known to many participants in this study, with many thinking that either masks or gloves would be sufficient to stop the spread. Our qualitative coding provided evidence of this. The primary concern of public health authorities is that should people wear masks, they may not practise more effective preventive behaviours such as hand hygiene and physical distancing because they believe masks and gloves are enough to protect them from COVID-19. To improve public health messaging, individual behaviour change techniques such as teaching prompts and cues ${ }^{14}$ about how to take masks off and to wash hands after touching a mask, would address the impression that masks alone were sufficient.

Overall, our findings are concordant to those of a recent online survey of over 4000 Australians which also found gaps in understanding. McCaffery $e t a l^{7}$ found that those with inadequate health literacy and who spoke a language other than English were less able to identify behaviours to prevent infection and experienced more difficulty finding information and understanding public health messaging about COVID-19. This study also identified that, there were higher endorsements of misinformation including negative views of vaccines among the same groups. ${ }^{7}$ Given that these views have broader public health implications (decreased vaccine uptake, increased antibiotic resistance), it is critical that we target future messaging to disadvantaged populations such as those with low health literacy and those who are culturally and linguistically diverse to ensure that health consumers are better equipped to make informed decisions with regard to their healthcare.

Finally, to develop effective prevention messages, consideration should be given to where individuals access COVID-19-related information. Counterintuitively, less than a quarter of participants nominated social media platforms in their top three sources of COVID-19 information. Most participants nominated commercial and government sponsored news channels as their top sources. Combining behaviour change techniques in prevention behaviours and promoting these messages in the most accessible and widely used media platforms are critical to ensure continued public cooperation in practising COVID-safe behaviours.

\section{Strengths and limitations}

In our sample, $70 \%$ of participants were born in Australia. Although this reflects Australian population demographics, results from this survey may not translate to individuals where English is not their primary language. While we assessed readability of the survey, we only informally assessed comprehension when we pilot tested the survey with community members and colleagues. We also did not assess cultural and linguistic diversity of the survey which limits the generalisability of our findings to this group. McCaffery $e t a l^{7}$ identified that participants whose primary language was not English had poorer understanding of COVID-19 symptoms, were less able to identify behaviours that reduced infection risk and experienced more difficulty understanding government messaging. Public health messaging not only should ensure translation of messages but also understand the cultural differences that may interfere with practising preventive behaviours. Unlike other Australian surveys, ${ }^{58}$ our survey looked beyond quantifying knowledge and attitudes to include qualitative content analyses to identify specific reasons for participants' misconceptions.

Our sample was balanced for gender and stratified for age. We also had proportionally representative quotas from each Australian state and territory. Despite these efforts, we acknowledge that survey research (with panel providers) has certain biases such as selection and sampling bias, and therefore suggest care when interpreting any results.

\section{CONCLUSION}

Our results suggest that although clear public messaging about the two key evidence-based prevention behaviours (hand hygiene and physical distancing) is broadly understood, there are still important knowledge gaps around how the disease is prevented, transmitted and its symptoms. Public health messages will need to combat the misconceptions that antibiotics and complementary medicines are effective in prevention and/or treating COVID-19. These beliefs have the potential to impede individuals from practising appropriate and effective behaviours. In the absence of a vaccine or effective drug treatments, our only prevention strategies are non-drug interventions, especially physical distancing and hand hygiene. ${ }^{1}$ How do we maintain these behaviours in the long term and how do we initiate other behaviours yet to be seen as critical such as wearing masks, testing when 
symptomatic, self-isolating and downloading contact tracing apps? By understanding some of the misconceptions identified in our survey and using the principles of public communication and knowledge translation, we can develop intervention strategies for the longer term.

Twitter Rae Thomas @rthomasEBP and Tammy C Hoffmann @Tammy_Hoffmann Contributors RT and PG conceived the study. All authors equally contributed to the study design. RT, ZAM and EA conducted the literature and internet searches. All authors contributed to selection of survey items. $\mathrm{HG}$ drafted the initial survey with critical input from all coauthors. RT, HG, ZAM and EA conducted the descriptive analyses. RT and LH conducted the qualitative analyses. RT drafted the initial manuscript with critical input from all other coauthors.

Funding RT, HG and ZAM are supported by NHMRC Program grant (\#1106452). TH is supported by a NHMRC Research Fellowship (\#1154607). KM is supported NHMRC Research Fellowship (\#1121110). PG is supported by a NHMRC Research Fellowship (\#1080042).

Competing interests None declared.

Patient consent for publication Not required.

Ethics approval Bond University Human Research Ethics Committee provided ethics approval for this research (\#RT03008).

Provenance and peer review Not commissioned; externally peer reviewed.

Data availability statement Data are available upon reasonable request.

Supplemental material This content has been supplied by the author(s). It has not been vetted by BMJ Publishing Group Limited (BMJ) and may not have been peer-reviewed. Any opinions or recommendations discussed are solely those of the author(s) and are not endorsed by BMJ. BMJ disclaims all liability and responsibility arising from any reliance placed on the content. Where the content includes any translated material, BMJ does not warrant the accuracy and reliability of the translations (including but not limited to local regulations, clinical guidelines, terminology, drug names and drug dosages), and is not responsible for any error and/or omissions arising from translation and adaptation or otherwise.

Open access This is an open access article distributed in accordance with the Creative Commons Attribution Non Commercial (CC BY-NC 4.0) license, which permits others to distribute, remix, adapt, build upon this work non-commercially, and license their derivative works on different terms, provided the original work is properly cited, appropriate credit is given, any changes made indicated, and the use is non-commercial. See: http://creativecommons.org/licenses/by-nc/4.0/.

\section{ORCID iDs}

Rae Thomas http://orcid.org/0000-0002-2165-5917

Zoe A Michaleff http://orcid.org/0000-0002-0360-4956

Tammy C Hoffmann http://orcid.org/0000-0001-5210-8548

Kirsten McCaffery http://orcid.org/0000-0003-2696-5006

Paul Glasziou http://orcid.org/0000-0001-7564-073X

\section{REFERENCES}

1 Hoffmann T, Glasziou P. What if the vaccine or drugs don't save us? Plan B for coronavirus means research on alternatives is urgently needed. Online: The Conversation, 2020. Available: https:// theconversation.com/what-if-the-vaccine-or-drugs-dont-save-usplan-b-for-coronavirus-means-research-on-alternatives-is-urgentlyneeded-136833

2 Kwok YLA, Harris P, McLaws M-L. Social cohesion: the missing factor required for a successful hand hygiene program. Am J Infect Control 2017;45:222-7.
3 World Health Organisation Managing the COVID-19 infodemic. Promoting healthy behaviours and mitigating the harms from misinformation and disinformation, 2020. Available: https://www. who.int/news/item/23-09-2020-managing-the-covid-19-infodemicpromoting-healthy-behaviours-and-mitigating-the-harm-frommisinformation-and-disinformation

4 Bonell C, Michie S, Reicher S, et al. Harnessing behavioural science in public health campaigns to maintain 'social distancing' in response to the COVID-19 pandemic: key principles. J Epidemiol Community Health 2020;74:617-9.

5 Cane J, O'Connor D, Michie S. Validation of the theoretical domains framework for use in behaviour change and implementation research. Implement Sci 2012;7:37.

6 Bavel JJV, Baicker K, Boggio PS, et al. Using social and behavioural science to support COVID-19 pandemic response. Nat Hum Behav 2020;4:460-71.

7 McCaffery KJ, Dodd RH, Cvejic E, et al. Health literacy and disparities in COVID-19-related knowledge, attitudes, beliefs and behaviours in Australia. Public Health Res Pract 2020;30. doi:10.17061/phrp30342012. [Epub ahead of print: 09 Dec 2020].

8 Geldsetzer P. Knowledge and perceptions of COVID-19 among the general public in the United States and the United Kingdom: a crosssectional online survey. Ann Intern Med 2020;173:157-60.

9 Atchison CJ, Bowman L, Vrinten C. Perceptions and behavioural responses of the general public during the COVID-19 pandemic: a cross-sectional survey of UK adults. MedRxiv. doi:10.1101/2020.04.01.20050039

10 Seale $\mathrm{H}$, Heywood AE, Leask J, et al. COVID-19 is rapidly changing: examining public perceptions and behaviors in response to this evolving pandemic. PLoS One 2020;15:e0235112.

11 Wise T, Zbozinek TD, Michelini G, et al. Changes in risk perception and self-reported protective behaviour during the first week of the COVID-19 pandemic in the United States. $R$ Soc Open Sci 2020;7:200742.

12 Australian Bureau of Statistics. Australian Bureau of statistics, sample size calculator, 2020. Available: https://www.abs.gov.au/ websitedbs/d3310114.nsf/home/sample+size+calculator

13 Downe-Wamboldt B. Content analysis: method, applications, and issues. Health Care Women Int 1992;13:313-21.

14 Michie S, Atkins L, West R. The behaviour change wheel. A guide to designing interventions. 1st ED. great Britain: Silverback publishing, 2014: 1003-10.

15 Ventola CL. The antibiotic resistance crisis: part 1: causes and threats. P T 2015;40:3227-83.

16 McCullough AR, Parekh S, Rathbone J, et al. A systematic review of the public's knowledge and beliefs about antibiotic resistance. $J$ Antimicrob Chemother 2016;71:27-33.

17 Bakhit M, Del Mar C, Gibson E, Hoffmann T, et al. Exploring patients understanding of antibiotic resistance and how this may influence attitudes towards antibiotic use for acute respiratory infections: a qualitative study in Australian general practice. BMJ Open 2019;9:e026735.

18 Australian Government Services Australian. Medical benefits schedule, 2021. Available: http://medicarestatistics.humanservices. gov.au/statistics/mbs_item.jsp

19 Hartnett KP, Kite-Powell A, DeVies J, et al. Impact of the COVID-19 pandemic on emergency department visits - United States, January 1, 2019-May 30, 2020. MMWR Morb Mortal Wkly Rep 2020;69:699-704.

20 Lazzerini M, Barbi E, Apicella A, et al. Delayed access or provision of care in Italy resulting from fear of COVID-19. Lancet Child Adolesc Health 2020;4:e10-11.

21 Moynihan R, Sanders S, Michaleff ZA. Pandemic impacts on healthcare utilisation: a systematic review. MedRxiv 2020. doi:10.1101/2020.10.26.20219352

22 Elshaug A, Duckett S. Hospitals have stopped unnecessary elective surgeries - and shouldn't restart them after the pandemic, 2020. Available: https://theconversation.com/hospitals-have-stoppedunnecessary-elective-surgeries-and-shouldnt-restart-them-after-thepandemic-136259 